\title{
Surgical Management of Odontoid Fracture in Elderly
}

\author{
Muhammad Nadeem, ${ }^{1}$ Muhammad Jawad Noon, ${ }^{2}$ Rana Muhammad Omar Daraz Khan, ${ }^{2}$ Inayatuallah Khan' \\ 'Department of Neurosurgery, ${ }^{2}$ Medical student, Shifa International Hospital and Shifa College of Medicine, Islamabad, \\ Pakistan.
}

\section{ABSTRACT}

Odontoid type II fractures are associated with high mortality in the elderly. No formal guidelines are present regarding the treatment of such fractures. Their management can be done either conservatively or surgically, however, surgical treatment is technically demanding and relatively new.

We report a case of 75 years old man who presented to our clinical setting, following a history of fall. The CT scan and MRI showed odontoid type II fracture. With time, the neurological deficit got marked and he underwent transoral odontoidectomy and occipitocervical fixation using rods and screws via posterior approach. Patient's neurological condition improved dramatically and on the short term follow up, he had no motor deficit. Our experience suggests that occipitocervical fixation via posterior approach is a viable option for the management of odontoid type II fracture in the geriatric population.

Keywords: aged; odontoid process; spinal fractures.

\section{INTRODUCTION}

Odontoid fractures represent majority of cervical spine fractures in the elderly and of all the odontoid fractures, Anderson and D'Alonzo type II fractures are the most common. ${ }^{1,2}$ They are potentially life threatening thus, their appropriate management is necessary. In geriatric population, conservative treatment is associated with high risk of complications and lower fusion rate so surgical approach has recently been given consideration. $^{3}$

We report an extremely rare surgical procedure that combines transoral odontoidectomy and occipitocervical fixation, for the successful management of odontoid type II fracture in a 75 year old man. To our knowledge and literature search, this case report is unique and first of its kind from Pakistan.

\section{CASE REPORT}

A 75 year old man with one day history of fall, presented with severe neck pain. On examination he had restricted neck flexion, extension and lateral rotation. Rest of his neurologic examination was unremarkable. CT scan and MRI (Figure 1) of spine showed, odontoid type II fracture with posterior displacement of C1 arch, causing compression of cervicomedullary junction along with disruption of surrounding ligaments and a small hematoma just posterior to odontoid. The CT scan and MRI films were reviewed with the patient and his family. The pros and cons of both, conservative treatment as

Correspondence: Muhammad Jawad Noon, Shifa College of Medicine, Pitras Bukhari Road, H-8/4, Islamabad, 44000, Pakistan. Email: jawadnoon@gmail.com, Phone: +92 3336390754. 
well as surgical treatment were explained in detail. They did not agree for surgery. After a few hours, his oxygen saturation dropped and power in all his limbs reduced to $1 / 5$. Patient was rushed to the operating room, where transoral odontoidectomy was done to relieve the compression of brain stem and upper cervical cord. He was then shifted to surgical ICU and kept ventilated. The following day, occipitocervical fixation was done via posterior approach.

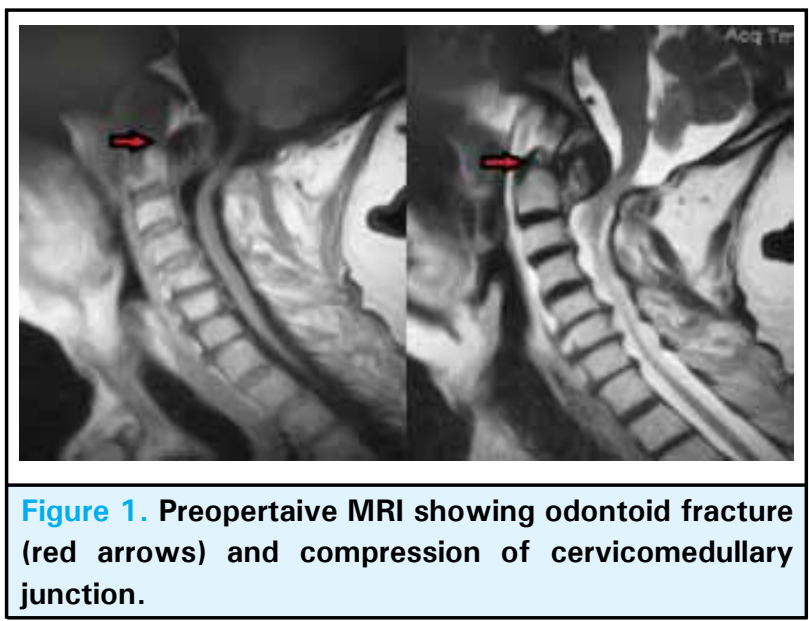

Surgical Technique: The patient was placed in supine position and underwent general anesthesia with orotracheal intubation without neck extension. After tracheostomy and removal of endotracheal tube, mouth was opened wide with Dingman's retractors. Soft palate was retracted with the help of foley's catheter. A vertical incision was made in posterior pharyngeal wall after identifying level with the help of C-Arm and pharyngeal muscles were retracted under microscope. Broken pieces of dens and anterior arch of atlas were removed until dura mater was seen adequately decompressed. Wound was then, closed in layers. The patient was shifted to surgical ICU and kept ventilated and relaxed. The next day, patient was again shifted to operating room and prone position was made very carefully in three pin head clamp. Occipitocervical fixation was done using screws in occipital bone, C2 pedicles and $\mathrm{C} 3, \mathrm{C} 4$ lateral masses (Figure 2). The screws were threaded to rods bilaterally. Bone graft was also placed after drilling outer cortex of laminae. The wound was closed in layers and patient's neck was immobilized in Philadelphia collar.

Post-operative recovery: Post operative CT scan showed improved alignment of the cervical spine and relief of compression effect on cord (Figure 3). Patient remained hemodynamically stable and could move all four limbs. The power of the limbs improved dramatically from $1 / 5$ to $4 / 5$ bilaterally and oxygen saturation came back to normal. Patient demonstrated progressive recovery and had no neurological deficit when he was discharged on $11^{\text {th }}$ post operative day. He was advised to wear cervical collar when mobilizing. Further follow up is awaited.
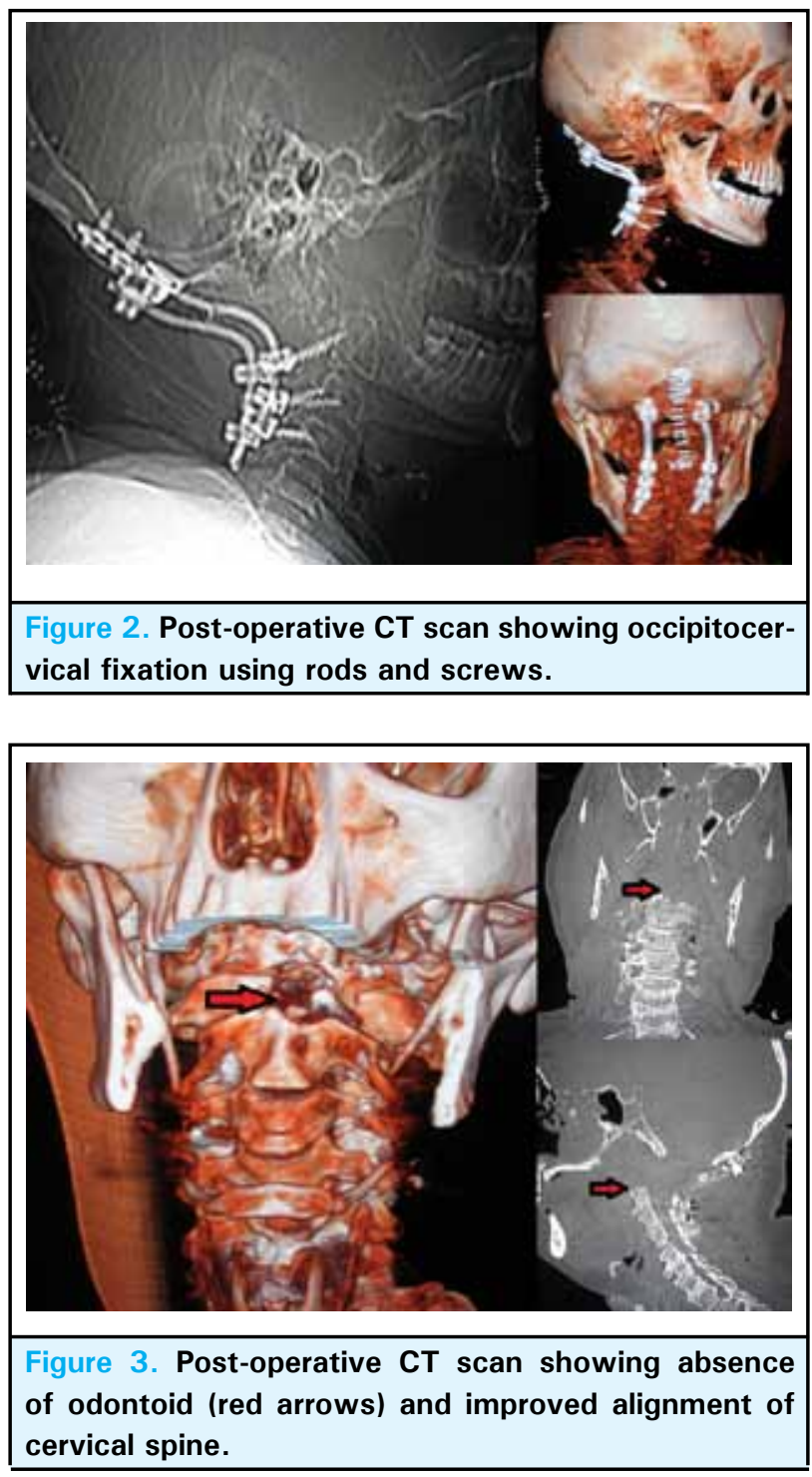

Follow-up: On one week follow up, the patient had no motor deficit and the power in all four of his limbs was $5 / 5$. Further follow up is awaited.

\section{DISCUSSION}

Odontoid type II fractures occur at the base of odontoid between the level of transverse ligament and the body of axis, as classified by Anderson and D'Alonzo. ${ }^{2}$ As demonstrated in our patient, they generally occur as a result of low energy fall, in elderly and are considered relatively unstable with highest nonunion rate. $^{1,4}$ Increased morbidity and mortality have been documented by a number of studies involving odontoid fractures in older patients. ${ }^{4-6}$ 
The treatment options for such fractures include conservative treatment; which constitutes the use of either cervical collar or halo vest, and surgical treatment; which involves either anterior screw fixation or posterior instrumentation. Due to paucity of data there is a debate over the best treatment choice in geriatric population and no formal guidelines have been devised.

Conservative treatment, although less challenging, is associated with high morbidity, mortality and nonunion rate. ${ }^{5,7}$ Majercik et al compared the use of halo vest in elderly to a 'death sentence' ${ }^{6}$ The complications include pin-track infection, CSF fluid leakage, higher risk of pneumonia and cardiac arrest. ${ }^{3,5,6}$

Surgical management of odontoid fracture is technically demanding due to the complex anatomy of the region but has recently been considered effective. Anterior screw fixation is recommended for the management of odontoid type II fractures, however, it is related with healing disturbances and other technical problems, in patients over 65 years of age. ${ }^{7,8}$ In contrast, posterior instrumentation is associated with fewer complications ${ }^{8}$ and $100 \%$ fusion rate can be achieved. ${ }^{9}$ Presently several techniques are being used for posterior instrumentation which include rod/wires, rod/screws, onlay/wires and plate/screws. We opted to do posterior instrumentation using rods and screws. A few small scale studies from developed countries show excellent success rate of this procedure and we had similar experience at our setting. ${ }^{9,10}$ In addition, we did not encounter any perioperative or postoperative complications.

Despite of the high difficulty level of the surgical procedure and anatomical challenges, we present a case of successful surgical management of odontoid type II fracture in an elderly that has not yet been reported from Pakistan. Our experience suggests that occipitocervical fixation via posterior approach can be considered as a viable option for the management of odontoid type II fracture in geriatric population however, there is a need of large scale studies before such recommendation can be made. Another limitation of the study is that, our observations are based on short term follow up. Large scale studies with long term follow up should be done before a definitive conclusion can be reached.

\section{REFERENCES}

1. Greene KA, Dickman CA, Marciano FF, Drabier JB, Hadley MN, Sonntag VK. Acute axis fractures: analysis of management and outcome in 340 consecutive cases. Spine. 1997;22(16):1843-52.

2. Anderson LD, D'Alonzo RT. Fractures of the odontoid process of the axis. J Bone Joint Surg Am. 1974;56(8):1663-74.

3. Glaser JA, Whitehill R, Stamp WG, Jane JA. Complications associated with the halo-vest. J Neurosurg. 1986;65(6):762-9.

4. Muller EJ, Wick M, Russe O, Muhr G. Management of odontoid fractures in the elderly. Eur Spine J. 1999;8(5):360-5.

5. Tashjian RZ, Majercik S, Biffl WL, Palumbo MA, Cioffi WG. Halo-vest immobilization increased early morbidity and mortality in elderly odontoid fractures. J Trauma. 2006;60(1):199-203.
6. Majercik S, Tashjian RZ, Biffl WL, Harrington DT, Cioffi WG. Halo vest immobilization in the elderly: a death sentence? J Trauma. 2005;59(2):350-7.

7. Börm W, Kast E, Richter HP, Mohr K. Anterior screw fixation in type II odontoid fractures: is there a difference in outcome between age groups? Neurosurgery. 2003;52(5):1089-94.

8. Andersson S, Rodrigues M, Olerud C. Odontoid fractures: high complication rate associated with anterior screw fixation in the elderly. Eur Spine J. 2000 Feb;9(1):56-9.

9. Harms J, Melcher RP. Posterior C1-C2 fusion with polyaxial screw and rod fixation. Spine. 2001;26(22):2467-71.

10. Abumi K, Takada T, Shono Y, Kaneda K, Fujiya M. Posterior occipitocervical reconstruction using cervical pedicle screws and plate-rod systems. Spine. 1999;24(14):1425-34. 